\title{
Mielolipoma adrenal bilateral em um cão
}

\author{
Bilateral adrenal myelolipoma in a dog
}

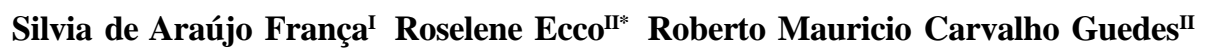

\begin{abstract}
O presente relato descreve um caso de RESUMO mielolipoma adrenal bilateral em uma cadela Poodle de 13 anos de idade. O cão morreu por peritonite séptica aguda, decorrente de ulceração e perfuração intestinal por corpo estranho linear. O neoplasma foi achado incidentalmente durante a necropsia. Macroscopicamente, as adrenais estavam redondas e com volume e aumentados. Ao corte elas eram friáveis, vermelho-amarronzadas, intercaladas por áreas branco-amareladas levemente proeminentes. Histologicamente, extensas áreas do parênquima continham adipócitos bem diferenciados, células hematopoéticas e macrófagos com hemossiderina, achados característicos de mielolipoma adrenal.

Palavras-chave: doenças de cães, glândula adrenal, mielolipoma, oncologia, patologia.
\end{abstract}

\section{ABSTRACT}

A case of bilateral adrenal myelolipoma in a 13year-old female Poodle is reported. Acute septic peritonitis due to intestinal ulceration and perforation caused by a linear foreign body was the cause of death. The adrenal neoplasm was an incidental necropsy finding. The adrenal glands were grossly rounded, enlarged and friable, and had red-brownish parenchyma interwoven by slightly prominent white-yellowish foci. Histologically, the findings of differentiated adipocytes in large areas of the parenchyma, in association to hematopoietic cells and macrophages with hemosiderin were consistent with the diagnosis of adrenal myelolipoma.

Key words: diseases of dogs, adrenal glands, myelolipoma, oncology, pathology.
O mielolipoma de adrenal é um neoplasma benigno constituído por tecido adiposo unilocular bem diferenciado e quantidades variáveis de células hematopoéticas. Esse tumor é considerado incomum em medicina veterinária e tem sido ocasionalmente descrito em cães (ZIMMER \& STAIR, 1983; NEWMAN et al., 2000; TURSI et al., 2005), gatos (GOURLEY et al., 1971; SANDER \& LANGHAM, 1972; McCAW et al., 1990) felídeos, em especial guepardos (CARDY \& BOSTROM, 1978) e aves exóticas (LATIMER \& RAKICH, 1995). As localizações mais freqüentes incluem o baço (SANDER \& LANGHAM, 1972; CARDY \& BOSTROM, 1978; ZIMMER \& STAIR, 1983), o fígado (GOURLEY et al., 1971; McCAW et al., 1990), o tecido subcutâneo (LATIMER \& RAKICH, 1995), o canal vertebral (NEWMAN et al., 2000) e as glândulas adrenais (TURSI et al., 2005). Um mielolipossarcoma foi relatado em uma ave silvestre (Perodicticus potto) originária da África tropical (LATIMER \& RAKICH, 1995).

O mielolipoma de adrenal é um neoplasma endocrinologicamente inativo e geralmente não está associado a disfunções endócrinas. Quase sempre o mielolipoma de adrenal se constitui em um achado incidental de necropsia, tanto em humanos quanto em animais (LAM \& LO, 2001; RÉPÁSSY et al., 2001; NAKAJO et al., 2003; MANASSERO et al., 2004). Em humanos, em alguns casos, o diagnóstico é feito por imagem, como ultra-sonografia, tomografia

\footnotetext{
IDepartamento de Medicina Veterinária, Faculdade de Castelo (FACASTELO), Castelo, ES, Brasil.

IISetor de Patologia Veterinária, Departamento de Clínica e Cirurgia Veterinária, Universidade Federal de Minas Gerais (UFMG), Belo Horizonte, MG, Brasil. E-mail: ecco@vet.ufmg.br. *Autor para correspondência.
} 
computadorizada e ressonância magnética (OTAL et al., 1999; NAKAJO et al., 2003; ELSAYES et al., 2004; PEREIRA et al., 2005). Essas técnicas também são utilizadas em clínica médica veterinária, entretanto, bem menos disponíveis em muitos locais (LATIMER \& RAKICH, 1995; MYERS, 1997).

O objetivo deste trabalho é descrever um caso de mielolipoma adrenal bilateral em uma cadela Poodle de 13 anos de idade. O cão foi encaminhado ao hospital veterinário com histórico clínico de tosse, anorexia, adipsia, prostração e vômito, e morreu logo após a internação. À necropsia constatou-se, como causa da morte, peritonite séptica aguda decorrente de ulceração e perfuração intestinal por corpo estranho linear.

Macroscopicamente, as glândulas adrenais estavam redondas e aumentadas, com aproximadamente $2 \mathrm{~cm}$ de diâmetro. Nas superfícies de corte, não havia diferenciação córtico-medular. Observavam-se áreas vermelho-amarronzadas entremeadas por áreas lobulares amareladas com cerca de $0,8 \mathrm{~cm}$ de diâmetro, bem delimitadas e friáveis (Figura 1A). Amostras teciduais das adrenais foram coletadas, fixadas em formalina a $10 \%$ tamponada e processadas pela técnica rotineira de inclusão em parafina e coloradas pela hematoxilina \& eosina.

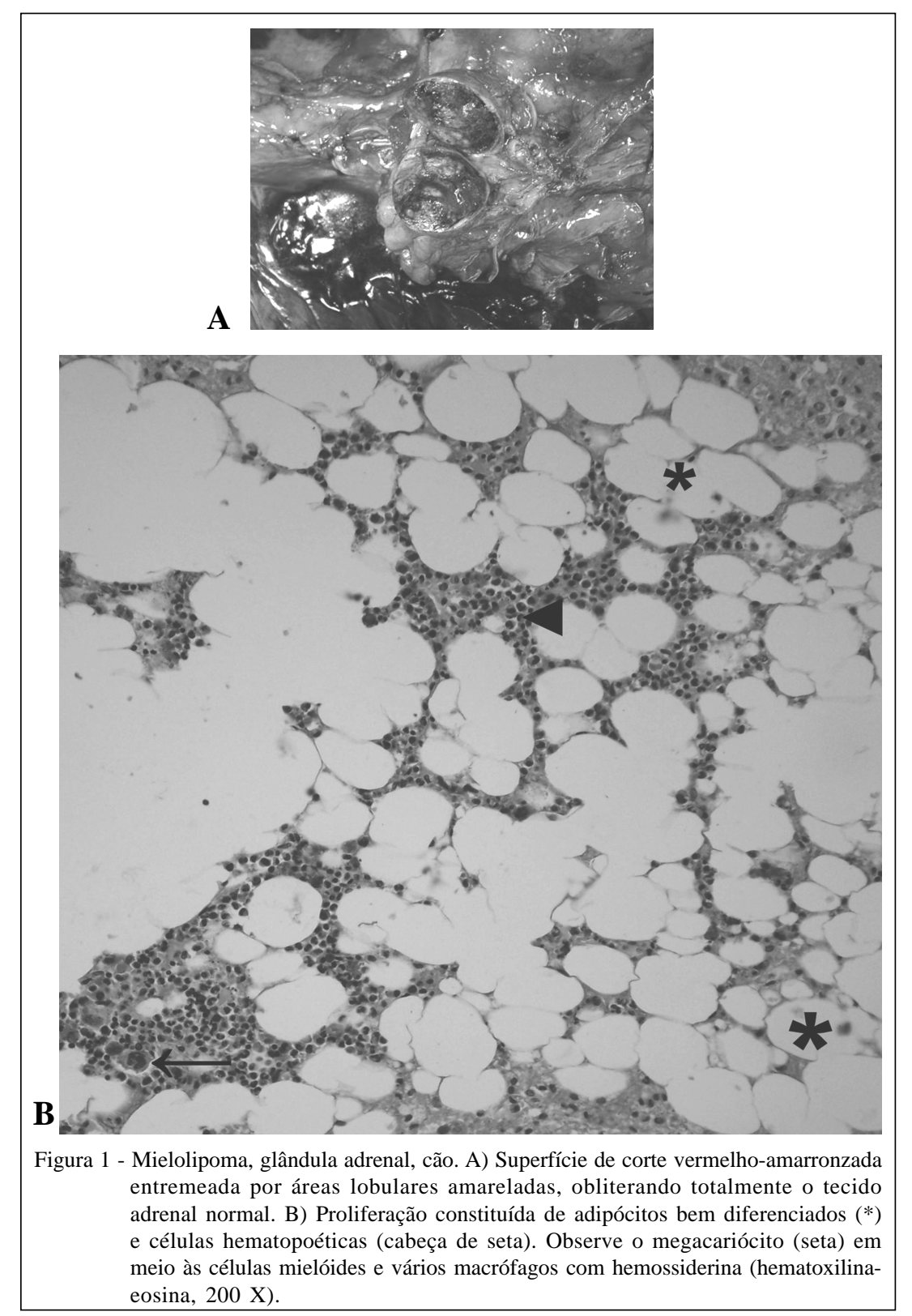

Ciência Rural, v.38, n.5, ago, 2008. 
$\mathrm{Na}$ histologia, ambos os neoplasmas adrenais demonstraram áreas multifocais constituídas por adipócitos bem diferenciados intercalados, por grupos de células hematopoéticas (Figura 1B), das linhagens mielóide e linfóide, em diferentes estágios de maturação. Em alguns locais visualizavam-se agregados de linfócitos e plasmócitos. Havia ainda numerosos vasos com trombos, áreas multifocais de hemorragia, necrose, focos de mineralização, alguns neutrófilos e numerosos macrófagos com citoplasma repleto de hemossiderina, confirmadas pela coloração especial azul da Prússia. Havia áreas onde o neoplasma permanecia separado da zona glomerular por uma faixa estreita de colágeno. Em alguns locais a zona fasciculada permanecia preservada e havia grupos de adipócitos e células hematopoéticas entre essas células. Havia também áreas de hiperplasia nodular cortical.

Embora a patogênese exata do mielolipoma seja desconhecida, acredita-se que o neoplasma possa ser decorrente de metaplasia adrenocortical, embolismo de medula óssea ou desenvolvimento de remanescentes embrionários presentes no parênquima da adrenal (NEWMAN et al., 2000; RÉPÁSSY et al., 2001; MANASSERO et al., 2004).

A maioria dos mielolipomas adrenais são pequenos e sem significado clínico. No entanto, há casos descritos em humanos em que o tratamento cirúrgico é recomendado pelo risco de ruptura espontânea e hemorragia (MANASSERO et al., 2004). Compressão dos órgãos adjacentes e dor abdominal também foram observadas (RÉPÁSSY et al., 2001). Mielolipomas são altamente vascularizados e, por isso, recomenda-se hemostasia meticulosa durante a excisão cirúrgica e monitoramento constante do paciente para choque hipovolêmico (LATIMER \& RAKICK, 1995).

O diagnóstico diferencial do mielolipoma nas adrenais abrange neoplasmas benignos e malignos. Os neoplasmas benignos primários da adrenal incluem adenoma adrenocortical, feocromocitoma e teratoma; tumores malignos incluem carcinoma cortical e lipossarcoma (LATIMER \& RAKICH, 1995; RÉPÁSSY et al., 2001; NAKAJO et al., 2003; PEREIRA et al., 2005). Em humanos, os neoplasmas metastáticos adrenais são os mais freqüentes, especialmente carcinomas pulmonares e mamários. Esta alta incidência pode ser explicada pelo rico suprimento de sinusóides sangüíneos das glândulas adrenais. No entanto, a real freqüência em animais é pouco conhecida. Um estudo retrospectivo relatou metástase na adrenal em $21 \%$ dos animais com neoplasmas disseminados. Dentre eles, linfoma, hemangiossarcoma e carcinomas mamários foram os mais freqüentes. Histologicamente, a infiltração de células neoplásicas se dissemina pelo córtex, ao longo de sinusóides vasculares, assim como ocorre em humanos (LABELLE \& DE COCK, 2005). Mielolipoma adrenal associado com adenoma cortical foi relatado em humanos (MASSANERO et al., 2004). Esta associação não foi encontrada na literatura médica veterinária. A ocorrência bilateral do mielolipoma na glândula adrenal é ainda mais rara (LATIMER \& RAKICH, 1995; NEWMAN et al., 2000).

Histologicamente deve-se diferenciar mielolipoma de eritropoese extramedular (NEWMAN et al., 2000; MANASSERO et al, 2004; PEREIRA et al., 2005). O adenoma adrenocortical é parcial ou completamente encapsulado e constituído por células bem diferenciadas, semelhantes às células secretoras das zonas fasciculada e reticulada (CAPEN, 2002). A eritropoese extramedular apresenta limites imprecisos, a celularidade é maior e não há acúmulo significativo de tecido adiposo (LATIMER \& RAKICH, 1995). Agregados de linfócitos são comumente descritos em mielolipomas e são encontrados com menos freqüência na eritropoese extramedular (McCAW et al., 1990).

Como foi mencionado anteriormente, relatos de mielolipoma bilateral são raros. Foi encontrada apenas uma descrição desse neoplasma com localização na adrenal em cães. É importante lembrar que o exame histológico é fundamental para o diagnóstico diferencial. Nesses casos, a presença de adipócitos, e células hematopoéticas são consistentes com o diagnóstico de mielolipoma da adrenal.

\section{REFERÊNCIAS}

CAPEN, C.C. Tumors of the endocrine glands. In: MEUTEN, D.J. (Ed.). Tumors in domestic animals. 4 ed. Ames: Iowa State, 2002. p.607-696.

CARDY, R.H.; BOSTROM, R.E. Multiple splenic myelolipoma in a cheetah (Acinonyx jubatus). Veterinary Pathology, v.15, p.556-558, 1978.

ELSAYES, K.M. et al. Adrenal masses: MR imaging features with pathologic correlation. Radiographics, v.24, p.573586, 2004

GOURLEY, I.M. et al. Myelolipomas of the liver in a domestic cat. Journal American Veterinary Medical Association, v.158, p.2053-2057, 1971.

LABELLE, P.; DE COCK, H.E.V. Metastatic tumors to the adrenal glands in domestic animals. Veterinary Pathology, v.42, p.52-58, 2005

LAM, K.Y.; LO, C.Y. Adrenal lipomatous tumours: a 30 year clinicopathological experience at a single institution. Journal of Clinical Pathology, v.54, p.707-712, 2001. 
LATIMER, K.S.; RAKICH, P.M. Subcutaneous and hepatic myelolipomas in four exotic birds. Veterinary Pathology, v.32, p.84-87, 1995.

MANASSERO, F. et al. Adrenal myelolipoma associated with adenoma. International Journal of Urology, v.11, p.326328, 2004.

MCCAW, D.L. et al. Hepatic myelolipoma in a cat. Journal American Veterinary Medical Association, v.197, p.243244, 1990.

MYERS, N.C. Adrenal incidentalomas. Veterinary Clinics of North America: Small Animal Practice, v.27, p.381399, 1997.

NAKAJO, M. et al. Embolization for spontaneous retroperitoneal hemorrhage from adrenal myelolipoma. Radiation Medicine, v.21, p.214-219, 2003.

NEWMAN, S.J. et al. Extradural myelolipoma in a dog. Journal Veterinary Diagnostic Investigation, v.12, p.71-74, 2000.
OTAL, P. et al. Imaging features of uncommon adrenal masses with histopathologic correlation. Radiographics, v.19, p.569581, 1999.

PEREIRA, J.M. et al. CT and MR imaging of extrahepatic fatty masses of the abdômen and pelvis: Techniques, diagnosis, diferencial diagnosis, and pitfalls. Radiographics, v.25, p.6985, 2005.

RÉPÁSSY, D.L. et al. Giant adrenal myelolipoma. Pathology Oncology Research, v.7, p.72-73, 2001.

SANDER, C.H.; LANGHAM, R.F. Myelolipoma of the spleen in a cat. Journal of the American Veterinary Medical Association, v.160, p.1101-1103, 1972.

TURSI, M. et al. Adrenal myelolipoma in a dog. Veterinary Pathology, v.42, p.232-235, 2005.

ZIMMER, M.A.; STAIR, E.L. Splenic myelolipoma in two dogs. Veterinary Pathology, v.20, p.637-638, 1983. 